Diabetologe 2011 7 7:300-300

DOI 10.1007/s11428-011-0695-y

Online publiziert: 9. Juli 2011

(c) Springer-Verlag 2011

\section{J.J. Meier}

Abteilung für Diabetologie und Gastrointestinale Endokrinologie, Medizinische Klinik I, Universitätsklinikum St. Josef-Hospital, Bochum

\title{
Inkretinbasierte Therapien auf dem Vormarsch
}

Seit ca. 4 Jahren stehen in Deutschland die inkretinbasierten Therapien in Form der „Glucagon-like peptide 1“-Analoga (GLP1-Analoga, Inkretinmimetika) und Dipeptidylpeptidase- (DPP-)4-Hemmer zur Verfügung. Die aktuell zugelassenen Präparate sind aufseiten der GLP-1-Analoga Exenatid und Liraglutid; bei den DPP-4Hemmern sind Sitagliptin, Vildagliptin und Sagaliptin zu nennen. Weitere Präparate (Exenatide-LAR, Linagliptin etc.) werden wahrscheinlich in Kürze folgen.

Wenngleich die hohen Kosten der inkretinbasierten Präparate eine sorgfältige Abwägung ihrer Verwendung im Vergleich zu etablierten Therapieformen erfordern, zeigte sich dennoch in den vergangenen Jahren eine kontinuierliche Zunahme des Einsatzes dieser Präparate in der klinischen Praxis. Dies begründet sich insbesondere durch die offensichtlichen Vorteile hinsichtlich des Risikos für Hypoglykämien und der Entwicklung des Körpergewichts im Vergleich zu etablierten blutzuckersenkenden Therapieansätzen.

In der aktuellen Ausgabe von Der Diabetologe führt Prof. Gallwitz zunächst in systematischer Weise durch das zunehmende Dickicht der jeweiligen Charakteristika, Indikationen sowie Sicherheitsund Verträglichkeitsprofile der derzeit verfügbaren inkretinbasierten Therapien. Auch werden die wesentlichen Unterschiede und Differenzierungsmerkmale der DPP-4-Hemmer und GLP-1-Analoga einander gegenübergestellt. Die in der Praxis dominierende Fragen, welche Patienten am ehesten von einer inkretinbasierten Therapie profitieren und welche Faktoren eher gegen ihren Einsatz sprechen, werden von Herrn Dr. Merker in seiner Übersicht aufgegriffen. Auch finden sich wichtige gesundheitsökonomische Hinweise zur Verordnungsfähigkeit der Präparate vor dem Hintergrund der aktuellen Restriktionen im Gesundheitswesen. Große Hoffnungen wurden zum Zeitpunkt der Markteinführung der GLP-1-basierten Therapien anhand verschiedenster tierexperimenteller Untersuchungen mit der Beobachtung einer Regeneration insulinproduzierender $\beta$-Zellen verbunden. Nach mehreren Jahren der klinischen Anwendung der GLP-1-Analoga und DPP-4-Hemmer werden diese initialen Erwartungen allerdings zunehmend relativiert. $\mathrm{Ob}$ und in welchem Umfang die tierexperimentellen und zellbasierten Beobachtungen zur $\beta$-Zell-Regeneration auf die praktische Therapie von Patienten mit Typ-2-Diabetes übertragen werden können, wird Herr Dr. Menge in seiner Übersicht diskutieren. Schließlich haben sich im Laufe der vergangenen Jahre vielfältige weitere neue Erkenntnisse hinsichtlich möglicher weiterer, über die eigentliche Blutzuckersenkung hinausgehender, Wirkungen der inkretinbasierten Therapien ergeben. Professor Ritzel wird daher in seinem Beitrag über aktuelle Entwicklungen und Befunde zu möglichen kardiovaskulären Wirkungen, neuroprotektiven Effekten sowie einer potenziellen adjuvanten Therapie GLP-1-basierter Präparate bei Patienten mit Typ-1-Diabetes informieren.

Vor dem Hintergrund der zunehmenden Verbreitung der DPP-4-Hemmer und GLP-1-Analoga in der Therapie von Patienten mit Typ-2-Diabetes sowie in Anbetracht der rasanten Weiterentwicklung der inkretinbasierten Therapien er- scheint eine fundierte Kenntnis ihrer Charakteristika, Einsatzgebiete und Differenzierungsmerkmale für den praktisch tätigen Diabetologen von essenzieller Wichtigkeit. Ich hoffe sehr, dass die vorliegenden Übersichtsbeiträge durch namhafte Experten auf diesem Weg hilfreich sein werden.
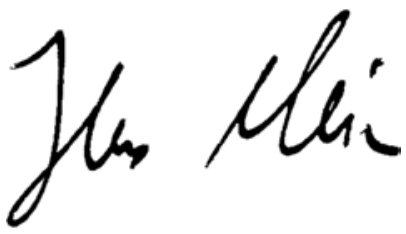

J.J. Meier

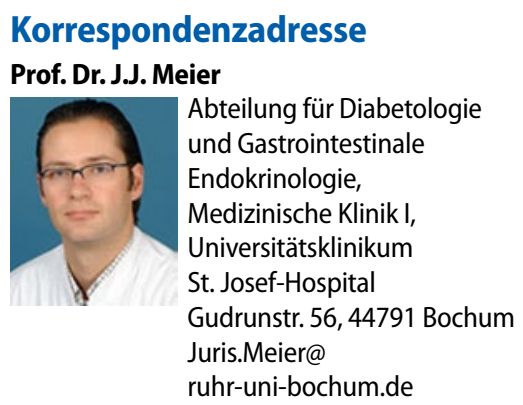

Interessenkonflikt. Der Autor weist auf folgende Beziehungen hin: Prof. Juris Meier hat im Rahmen von Vorträgen und Beratertätigkeiten Honorare von den folgenden Firmen erhalten: Astra Zeneca, Berlin-Chemie, BMS, Boehringer-Ingelheim, Eli Lilly, MSD, NovoNordisk, Novartis, Roche, Sanofi-Aventis. 